\title{
Two-dimensional Gallium and Indium Oxides from Global Structure Searching: Ferromagnetism and Half Metallicity via Hole Doping
}

\author{
Ruishen Meng ${ }^{1^{*}}$, Michel Houssa ${ }^{1^{*}}$, Konstantina Iordanidou ${ }^{2}$, Geoffrey Pourtois ${ }^{3}$, \\ Valeri Afanasiev ${ }^{l}$ and André Stesmans ${ }^{l}$ \\ ${ }^{1}$ Department of Physics and Astronomy, KU Leuven, Celestijnenlaan 200D, Leuven \\ B-3001, Belgium \\ ${ }^{2}$ Department of Physics, University of Oslo, NO-0316 Oslo, Norway \\ ${ }^{3}$ imec, Kapeldreef 75, B-3001 Leuven, Belgium \\ "Email: ruishen.meng@kuleuven.be; michel.houssa@kuleuven.be
}

\begin{abstract}
There has been tremendous research effort in hunting for novel two-dimensional (2D) materials with exotic properties, showing great promise for various potential applications. Here, we report findings about a new hexagonal phase of $2 \mathrm{D}_{\mathrm{Ga}_{2}} \mathrm{O}_{3}$ and $\mathrm{In}_{2} \mathrm{O}_{3}$, with high energetic stability, using a global searching method based on an evolutionary algorithm, combined with density-functional theory calculations. Their structural and thermal stabilities are investigated by the calculations of their phonon spectra and by ab-initio molecular dynamics simulations. They are predicted to be intrinsically non-magnetic stable semiconductors, with a flat band edge around the valence band top, leading to itinerant ferromagnetism and half-metallicity upon hole doping. Bilayer $\mathrm{Ga}_{2} \mathrm{O}_{3}$ is also studied and found to exhibit ferromagnetism without extra hole doping. The Curie temperature of these materials, estimated using Monte Carlo simulations based on the Heisenberg model, is around 40 to $60 \mathrm{~K}$ upon a moderate hole doping density.
\end{abstract}




\section{INTRODUCTION}

Computational screening of novel materials with intriguing properties has been attracting enormous research attention recently. ${ }^{1-4}$ Among this research, the prediction of new two-dimensional (2D) materials, especially those with magnetic properties, has also been widely explored..$^{5-8}$ These investigations have facilitated and accelerated the experimental discovery of materials for a variety of applications. Nevertheless, some of the predicted materials, found from property-oriented materials searching, may turn out to be unstable and very challenging to grow or synthesize. ${ }^{9-13}$ Therefore, stricter criteria are necessary for materials prediction, to make sure the candidate materials have global minimum of free energy, as well as high structural and thermal stabilities.

Various 2D ferromagnetic materials, for example, $\mathrm{CrX}_{3}(\mathrm{X}=\mathrm{Cl}, \mathrm{Br}, \mathrm{I}),{ }^{14-16}$ $\mathrm{Cr}_{2} \mathrm{Ge}_{2} \mathrm{Te}_{6},{ }^{16,17} \mathrm{Fe}_{3} \mathrm{GeTe}_{2}{ }^{18,19}$ monolayers, etc. have been theoretically predicted and experimentally synthesized. Further strategies such as electrostatic doping, electric field or strain effects, van der Waals stacking, have been intensively exploited to tune the magnetic properties of those monolayers. ${ }^{15,17,18,20-25}$ Apart from 2D materials with intrinsic magnetic properties, efforts have also been made to induce ferromagnetism in some nonmagnetic 2D semiconductors by e.g. hole doping. ${ }^{26-29}$ The spontaneous ferromagnetic order in those intrinsically nonmagnetic $2 \mathrm{D}$ materials originates from the exchange splitting of the electronic states at the top of the valence band, where a sharp Van Hove singularity exists in the density of states (DOS), leading to the Stoner instability. Therefore, these nonmagnetic 2D materials could also be promising for application in novel spintronic devices.

In this work, we explore all possible 2D phases of gallium oxides and indium oxides by means of the Universal Structure Predictor: Evolutionary Xtallography (USPEX) method, on the basis of first-principles calculations, to find out the most energetically stable atomic configurations, i.e. $\mathrm{M}_{2} \mathrm{O}_{3}(\mathrm{M}=\mathrm{Ga}$, In). Their stability is demonstrated by the formation energies, phonon dispersions as well as by molecular dynamics simulations. We find that $\mathrm{M}_{2} \mathrm{O}_{3}$ monolayers are nonmagnetic semiconductors, while hole doping can turn them into ferromagnetic materials over a wide range of hole 
concentrations. Their Curie temperatures $\left(T_{\mathrm{c}}\right)$ under different hole doping concentrations are also estimated.

\section{COMPUTATIONAL METHODS}

Searches for the possible structures of 2D gallium oxides and indium oxides, through an ab-initio evolutionary algorithm, was performed by USPEX, ${ }^{30,31}$ interfaced with the Vienna ab initio simulation package (VASP). ${ }^{32,33}$ The variable-composition searching was considered, with the total number of atoms in the 2D crystals fixed between 2 and 8 , and their vacuum spaces restricted to be within $20 \AA$, respectively. 40 groups of symmetries were used to produce a random symmetric structure generator for the initial population. Then, the full structure relaxations were performed, and the most stable and metastable atomic structures were screened and inherited into the next generation, by comparing their formation enthalpy. The population kept evolving until the most stable configuration stayed unchanged for a further 10 generations, in order to find the globally lowest energy state. All density functional theory (DFT) calculations were performed using VASP, with electron-ion interaction described by projector augmented wave (PAW) pseudopotentials. ${ }^{32}$ The generalized gradient approximation (GGA), parameterized by the Perdew-Burke-Ernzerhof (PBE) approach ${ }^{34}$, was used as the exchange correlation functional. The energy cutoff of $550 \mathrm{eV}$ and k-point meshes of $8 \times 8 \times 1$ and $32 \times 32 \times 1$, were used for structural optimizations and self-consistent calculations, respectively. A total energy convergence criterion of $10^{-5} \mathrm{eV}$ and a force convergence criterion of $0.005 \mathrm{eV} / \AA ̊$ were chosen for complete relaxations of the lattice constants and the atomic positions. The Heyd-Scuseria-Ernzerhof functional (HSE06), ${ }^{35}$ which mixes $25 \%$ nonlocal exchange with the PBE functional, was used for more accurate electronic band structure and density of states (DOS) calculations. Additionally, the optimized exchange van der Waals functional, optB86-vdW ${ }^{36}$, was used to take into account the van der Waals interactions in the calculations of the $\mathrm{Ga}_{2} \mathrm{O}_{3}$ bilayers.

Phonon dispersion curves were calculated by the PHONOPY package ${ }^{37}$ on the basis 
of Density Functional Perturbation Theory (DFPT). The ab-initio molecular dynamics (AIMD) simulations were carried out using $5 \times 5 \times 1$ supercells (with 125 atoms) and were equilibrated at $300 \mathrm{~K}$ for $10 \mathrm{ps}$ with a time step of $2 \mathrm{fs}$. The constant-temperature and volume canonical ensemble (NVT) was selected, and the temperature was controlled by means of a Nose thermostat. ${ }^{38,39}$

The Curie temperatures were estimated using Monte Carlo simulations, as implemented in the VAMPIRE package. ${ }^{40}$ Rectangular supercells of $100 \times 60 \sqrt{ } 3 \times 1$ were used for the simulations, since only orthogonal lattice vectors are supported. The spins were thermalized for 10000 equilibrium steps, followed by 20000 averaging steps for the calculation of the thermal equilibrium magnetization at every temperature.

\section{III.RESULTS AND DISCUSSION}

The convex hulls, defined as the enthalpy of formation versus the composition of gallium oxides and indium oxides with all possible stoichiometries, are plotted in the left panel of Fig .1 (a) and (b), respectively. The formation enthalpy per atom, $\triangle H$, which can be used to determine the relative stability between different compositions and structures, was calculated by

$$
\triangle H=\left(E_{M x O y}-x E_{M}-y E_{O 2} / 2\right) /(x+y)
$$

where $E_{M x O y}$ is the total energy of $M_{x} O_{y},(\mathrm{M}=\mathrm{Ga}, \mathrm{In}), E_{M}$, is the energy of the metal atom in its stable bulk structure, $E_{O 2}$ corresponds to the energy of an isolated oxygen molecule in its paramagnetic ground state. Any structure with its formation enthalpy on the convex hull is considered to be thermodynamically stable and experimentally synthesizable. The most stable structures of Ga-O and In-O, highlighted by the red dots in the convex hulls, are $\mathrm{Ga}_{2} \mathrm{O}_{3}$ and $\mathrm{In}_{2} \mathrm{O}_{3}$, respectively. Specifically, their $\triangle H$ can respectively reach $-1.95 \mathrm{eV} /$ atom and $-1.52 \mathrm{eV} /$ atom. In addition, the $\mathrm{GaS}$-like $\mathrm{GaO}$ monolayer is also found to be stable, since its formation enthalpy $(-1.65 \mathrm{eV} / \mathrm{atom})$ is on the convex hull. In this paper, we only focus on the most stable $\mathrm{M}_{2} \mathrm{O}_{3}$ monolayers. 

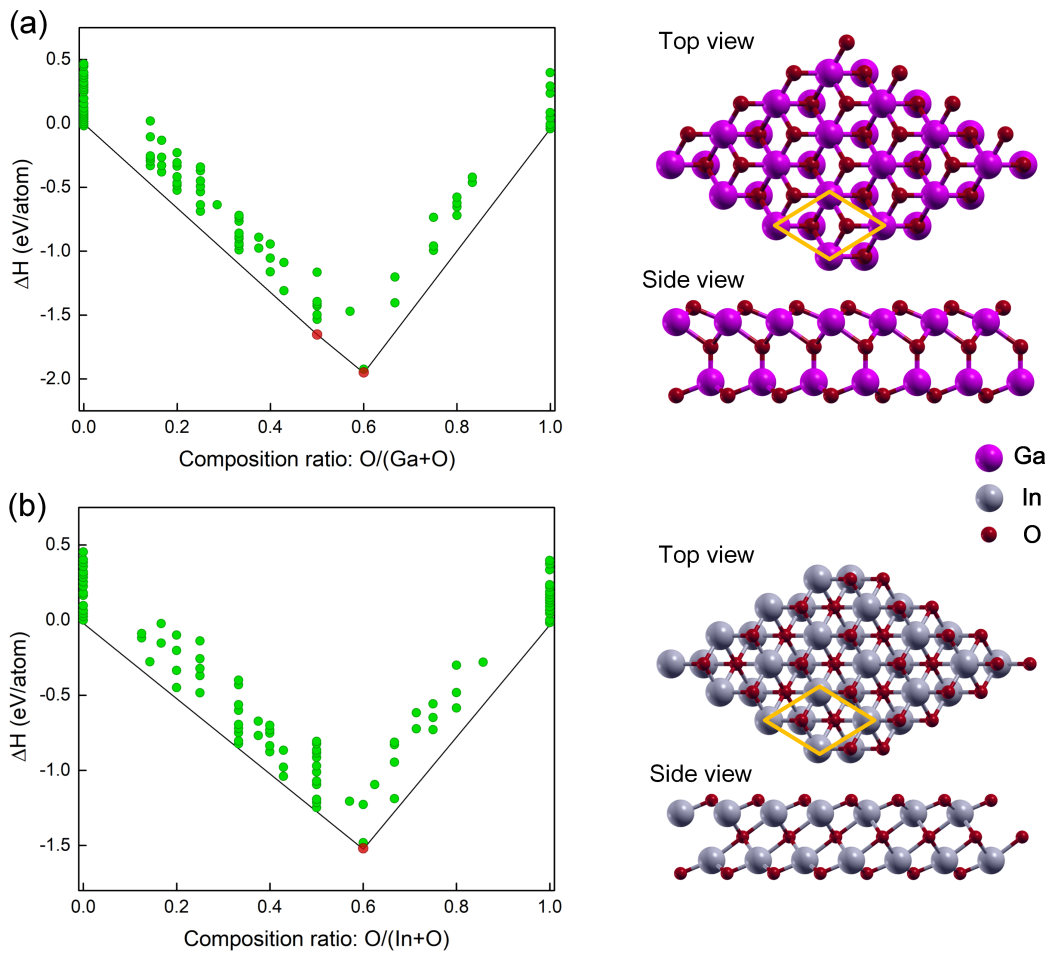

Fig. 1. Convex hulls of different stoichiometries searched by USPEX, and the corresponding atomic structures of the most energetically favorable candidates for $2 \mathrm{D}$ binary (a) Ga-O and (b) In-O materials. The red dots on the Convex hulls indicate the predicted stable phases. The unit cell is highlighted by yellow solid lines.

The relaxed crystal structures of single-layer $\mathrm{M}_{2} \mathrm{O}_{3}$ are given in the right panel of Fig. 1, which consist of five atomic sublayers stacked in the sequence of O-M-O-M-O in the out-of-plane direction. The calculated in-plane lattice constants of $\mathrm{Ga}_{2} \mathrm{O}_{3}$ and $\mathrm{In}_{2} \mathrm{O}_{3}$ monolayers are $3.10 \AA$ and $3.04 \AA$, respectively. The $\mathrm{Ga}_{2} \mathrm{O}_{3}$ monolayer is noncentrosymmetric (polar), similarly to $\operatorname{In}_{2} \mathrm{X}_{3}(\mathrm{X}=\mathrm{S}, \mathrm{S})$ monolayers. ${ }^{41,42}$ On the other hand, the $\mathrm{In}_{2} \mathrm{O}_{3}$ monolayer is found to be centrosymmetric (non-polar). The oxygen atoms on the top and bottom layer are bonded to three neighboring metal atoms, while the central oxygen atoms bond to 4 and 6 metal atoms for $\mathrm{Ga}_{2} \underline{\mathrm{O}}_{3}$ and $\operatorname{In}_{2} \underline{\mathrm{O}}_{3}$ monolayers, 
respectively. The indium atoms are 6-fold coordinated in $\mathrm{In}_{2} \mathrm{O}_{3}$, while in $\mathrm{Ga}_{2} \mathrm{O}_{3}$, the gallium atoms in the upper and lower layers are 6 and 4-fold coordinated, respectively.
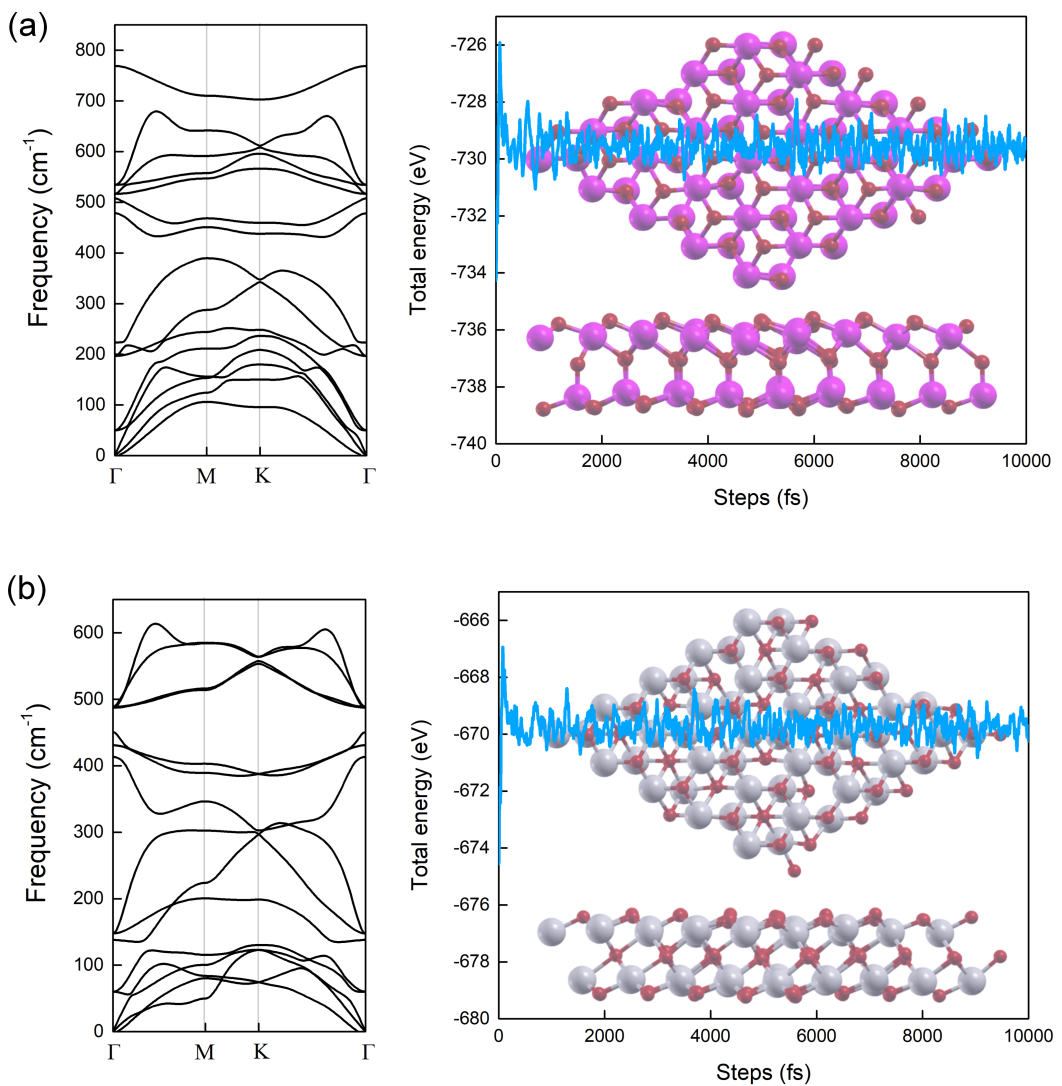

Fig. 2. Phonon dispersion spectra, and total energy evolution during molecular dynamics simulations, together with the atomic structures obtained after $10 \mathrm{ps}$, for (a) $\mathrm{Ga}_{2} \mathrm{O}_{3}$ and (b) $\mathrm{In}_{2} \mathrm{O}_{3}$ monolayers at $300 \mathrm{~K}$.

Phonon dispersion calculations, together with AIMD simulations were carried out to evaluate the dynamic stability as well as the room-temperature stability of the two $\mathrm{M}_{2} \mathrm{O}_{3}$ monolayers; the results are presented in Fig. 2. In the phonon dispersion spectra, merely no imaginary frequency is observed, which suggests that both $\mathrm{M}_{2} \mathrm{O}_{3}$ monolayers 
are dynamically stable. During the AIMD simulations, with the temperature fixed at $300 \mathrm{~K}$, the total energies of $\mathrm{M}_{2} \mathrm{O}_{3}$ monolayers oscillate within narrow ranges, i.e. $\sim 3$ eV/cell. In the addition, the atoms only slightly oscillate around their equilibrium positions, and the structural integrity is well retained after $10 \mathrm{ps}$. Given their low formation enthalpy, dynamical stability, together with their thermal stability, the growth of $\mathrm{M}_{2} \mathrm{O}_{3}$ monolayers appears highly possible.
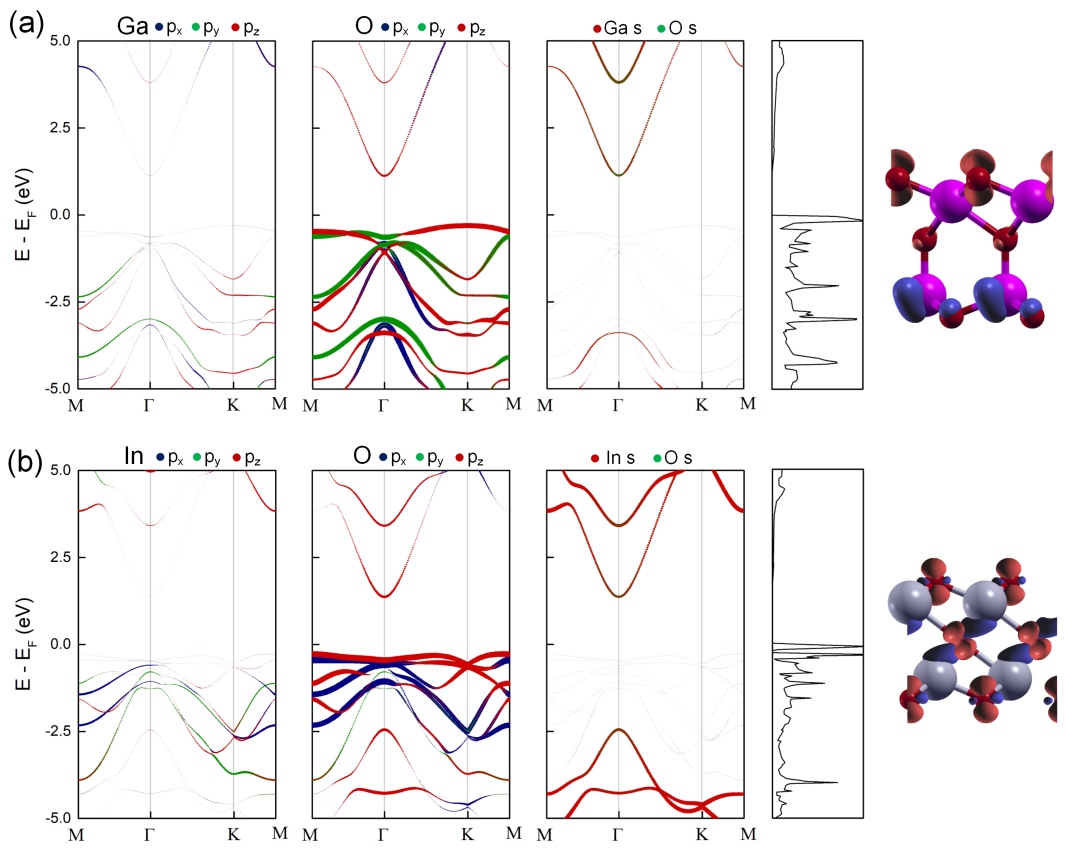

Fig. 3. Fat band structures, density of states and partial charge densities of the CBM (blue) and VBM (red) for (a) $\mathrm{Ga}_{2} \mathrm{O}_{3}$ and (b) $\mathrm{In}_{2} \mathrm{O}_{3}$ monolayers. The isosurface value is set to $0.2 \mathrm{e} / \AA^{3}$.

From the electronic band structures shown in Fig. 3, one can see that both $\mathrm{M}_{2} \mathrm{O}_{3}$ monolayers are semiconductors with indirect band gaps. Specifically, the band gap of $\mathrm{Ga}_{2} \mathrm{O}_{3}$ monolayer is $\sim 1.43 \mathrm{eV}$ at PBE level ( $2.72 \mathrm{eV}$ at HSE06 level, see Fig. S1); the bottom of the conduction band (CB) is contributed by the $s$ and $p_{z}$ orbital of oxygen 
atoms and $s$ orbitals of gallium atoms (denoted as O-s, O- $p_{z}, \mathrm{Ga}-s$, respectively), whereas the top of the valence band (VB) is mainly contributed by the O- $p_{y}$ and O- $p_{z}$ orbitals. In particular, the valence band maximum (VBM) essentially originates from the $p_{z}$ orbital of the top oxygen atoms, as clearly shown in the partial charge density plot. Concerning the $\operatorname{In}_{2} \mathrm{O}_{3}$ monolayer, the band gap calculated at PBE level is $\sim 1.64 \mathrm{eV}$ $(\sim 2.93 \mathrm{eV}$ at HSE06 level, see Fig. S1). The bottom CB originates mainly from the hybridization of O- $p_{z}$ and $\lfloor-s$ orbitals, while the top VB is merely derived from the O$p_{z}$ orbital. It is evident that the partial charge density of the VBM is only located on the oxygen atoms of the $\mathrm{In}_{2} \mathrm{O}_{3}$ monolayer.

(a)
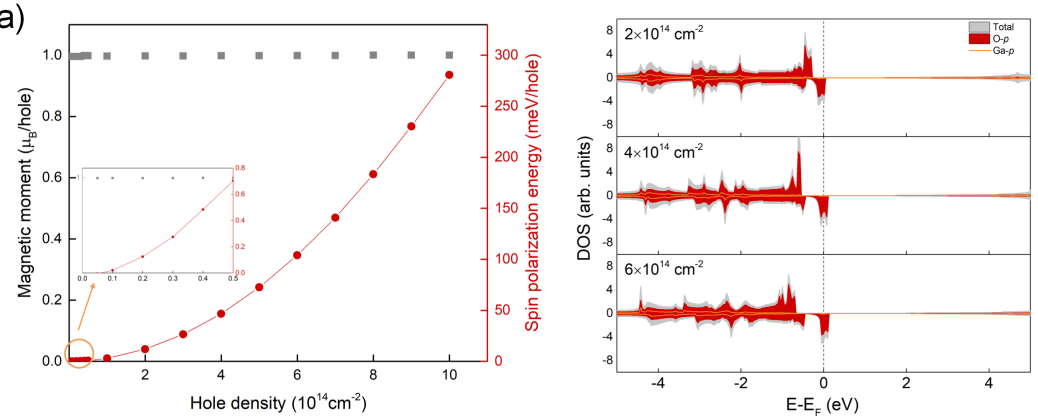

(b)
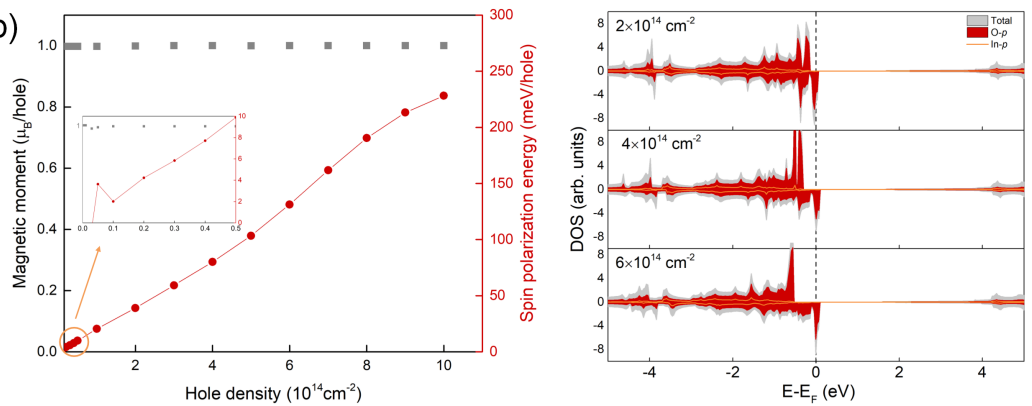

Fig. 4. Magnetic moment and spin polarization energy as a function of hole doping concentration, and spin-polarized partial density of states under different hole doping densities of (a) $\mathrm{Ga}_{2} \mathrm{O}_{3}$ and (b) $\mathrm{In}_{2} \mathrm{O}_{3}$ monolayers.

In the DOS of the $\mathrm{M}_{2} \mathrm{O}_{3}$ monolayers, there is a sharp peak in the edge of valence band. This sharp peak is related to so-called Van Hove singularities. When the Fermi 
level approaches the singularity, a transition from a paramagnetic phase to a ferromagnetic phase could occur, as reported in several previous works. ${ }^{26-29}$ Therefore, in the following, we will study hole doping of $\mathrm{M}_{2} \mathrm{O}_{3}$ monolayers, using spin-polarized DFT simulations, to investigate their possible magnetic properties.

By performing the relaxation of the charged cells, the effect of hole doping on the atomic structures is first investigated. The structural variation of the $\mathrm{M}_{2} \mathrm{O}_{3}$ monolayers upon hole doping is summarized in Fig. S2. The thickness of the $\mathrm{Ga}_{2} \mathrm{O}_{3}$ monolayer shrinks when it is hole doped, while for $\operatorname{In}_{2} \mathrm{O}_{3}$ monolayer, the thickness first decreases when the doping density is lower than $\sim 4 \times 10^{14} \mathrm{~cm}^{-2}$, then it increases and becomes larger than the original thickness before doping for a doping density higher than $\sim 7 \times 10^{14}$ $\mathrm{cm}^{-2}$. In general, the $\mathrm{M}_{2} \mathrm{O}_{3}$ monolayers do not suffer pronounced deformation upon hole doping, as the variation of the bond lengths and thicknesses is typically less than 5\%, even at a high hole doping concentration of $10^{15} \mathrm{~cm}^{-2}$, except for the In-In distance ( $~ 8 \%$ elongation) and a specific O-Ga bond length ( 12\% elongation), see Fig. S2. Therefore, we can conclude that hole doping has a limited effect on the atomic structures of the $\mathrm{M}_{2} \mathrm{O}_{3}$ monolayers.

The computed magnetic moments and spin polarization energies of $\mathrm{M}_{2} \mathrm{O}_{3}$ monolayers are shown in the left panel of Fig. 4, as a function of the hole doping density. The spin polarization energy is defined as the total energy of the non-magnetic state minus the one of the ferromagnetic state. Thus, a positive spin polarization energy indicates that the ferromagnetic state is more stable. The paramagnetic to ferromagnetic transition occurs at a hole concentration as low as $5 \times 10^{12} \mathrm{~cm}^{-2}$. In this case, the magnetic moment is $\sim 1.0 \mu_{\mathrm{B}} /$ hole (where $\mu_{\mathrm{B}}$ is the Bohr magneton), i.e. the injected holes are fully spin polarized. The spin polarization energy increases monotonously with the hole density, up to a hole concentration of $\sim 10^{15} \mathrm{~cm}^{-2}$ for both $\mathrm{M}_{2} \mathrm{O}_{3}$ monolayers.

The spin-polarized DOS of $\mathrm{M}_{2} \mathrm{O}_{3}$ monolayers under different doping levels are presented in the right panel of Fig. 4, where the energy splitting between spin up and spin down states can be observed. The Fermi level crosses the spin down states, while the up-spins are fully occupied at the Fermi level, making these two materials half- 
metallic upon hole doping. Obviously, the energy splitting increases with the increase of the hole density i.e., from $\sim 0.3 \mathrm{eV}(\sim 0.2 \mathrm{eV})$ at $2 \times 10^{14} \mathrm{~cm}^{-2}$, to $\sim 0.8 \mathrm{eV}(0.6 \mathrm{eV})$ at $6 \times 10^{14} \mathrm{~cm}^{-2}$ for $\mathrm{Ga}_{2} \mathrm{O}_{3}\left(\mathrm{In}_{2} \mathrm{O}_{3}\right)$ monolayer, respectively. In addition, the valence band top still mainly consists of the $p$ orbitals of the oxygen atoms when the hole doping concentration increases. Therefore, the oxygen atoms mostly contribute to the ferromagnetic order in the $\mathrm{M}_{2} \mathrm{O}_{3}$ monolayers.

(a)
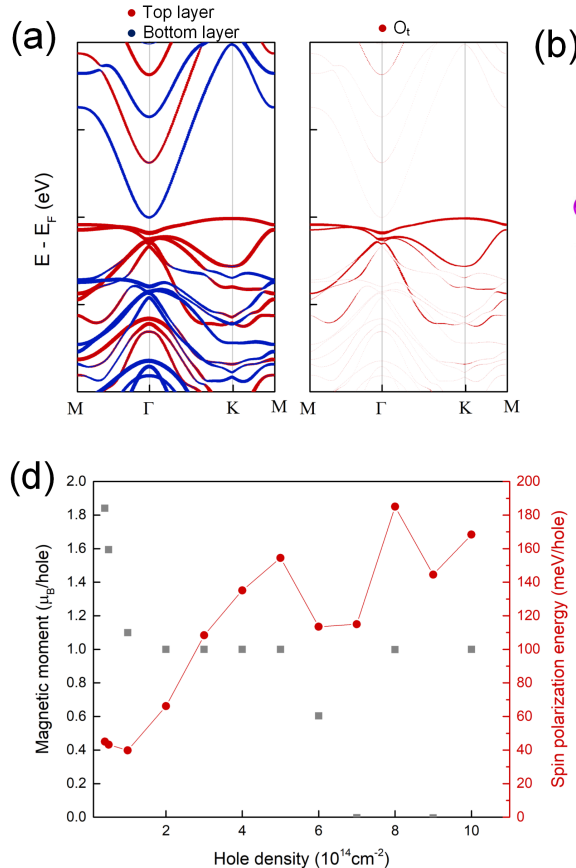

(b)

(c)
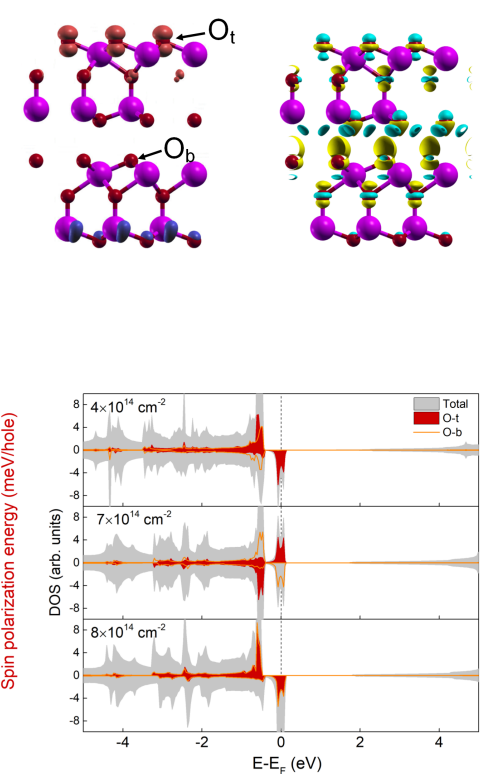

Fig. 5. (a) Fat band structures, (b) partial charge densities of the CBM (blue) and VBM (red), (c) charge density difference (Yellow and Cyan represents charge accumulation and depletion, respectively) (d) magnetic moment and spin polarization energy as a function of hole doping concentration, and spin-polarized partial density of states under different hole doping densities of a $\mathrm{Ga}_{2} \mathrm{O}_{3}$ bilayer. The isovalues of partial charge densities and charge density differences are fixed to $0.005 \mathrm{e} / \AA^{3}$ and $0.2 \mathrm{e} / \AA^{3}$, respectively. 
Similar to the situation in $\operatorname{In}_{2} X_{3}(X=S, S e)$ monolayers, the centrosymmetry of the $\mathrm{Ga}_{2} \mathrm{O}_{3}$ monolayer is broken, due to the different coordination environment of the $\mathrm{Ga}$ atoms, which results from the asymmetric positions of $\mathrm{O}$ atoms in the middle layer. This leads to an intrinsic dipole, pointing from the bottom to the top surface along the out of plane direction. It has been reported that the dipole moment will be almost doubled in $\operatorname{In}_{2} \mathrm{X}_{3}(\mathrm{X}=\mathrm{S}, \mathrm{Se})$ bilayers. ${ }^{42}$ Consequently, we also considered $\mathrm{Ga}_{2} \mathrm{O}_{3}$ bilayers to investigate the influence of this enhanced polarization on the magnetic properties. We constructed 6 different stacking patterns, as shown in Fig. S3, and the most stable one was studied further. Using the optB86-vdW functional and spin polarized calculations for the atomic relaxation, the interlayer distance is calculated to be $2.29 \AA$, and the lattice constant is $3.10 \AA$. Surprisingly, we found that the $\mathrm{Ga}_{2} \mathrm{O}_{3}$ bilayer exhibits ferromagnetism without hole doping, with a magnetic moment of $\sim 0.03$ $\mu_{\mathrm{B}}$ per unit cell, which is comparable to a hole doping density of $\sim 4 \times 10^{13} \mathrm{~cm}^{-2}$. To find out the underlying mechanism responsible for the spontaneous ferromagnetism, we computed the partial band structures, partial charge densities, as well as the charge density difference of the $\mathrm{Ga}_{2} \mathrm{O}_{3}$ bilayer, presented in Fig. 5. Clearly, the bottom CB of the double layer is solely contributed by the bottom layer, while the top VB is originating from the top layer, which gives rise to the typical type-II band alignment. In addition, the $\mathrm{CB}$ crosses the Fermi level, making the bilayer a metal. This is also confirmed by the HSE band structure calculations, as shown in Fig. S1 (c). On the other hand, the $p$ orbitals of the topmost oxygen atoms of the top layer $\left(\mathrm{O}_{\mathrm{t}}\right)$ mostly contribute to the top VB, as shown in the band structure; this is also confirmed by the partial charge density plot shown in Fig. 5 (b). Note that these topmost oxygen atoms mainly contribute to the magnetic moment of the $\mathrm{Ga}_{2} \mathrm{O}_{3}$ bilayers. The intrinsic dipole present in the bilayer is induced by electrons transferred from the top layer to the bottom layer, resulting in the "hole doping" of the top layer, and the occurrence of a ferromagnetic order in the material; this is also reflected by the charge density difference plot in Fig. 5 (c).

The magnetic properties of the $\mathrm{Ga}_{2} \mathrm{O}_{3}$ bilayer, induced by increasing further the hole 
doping density, was also investigated. The computed magnetic moment, shown in Fig. 5 (d), is larger than $1.0 \mu \mathrm{B} /$ hole, when the hole doping density is less than or equal to $1 \times 10^{14} \mathrm{~cm}^{-2}$, then it decreases gradually to $\sim 1.0 \mu_{\mathrm{B}} /$ hole when the doping density is further increased. Nevertheless, the magnetic moment gradually declines again when the hole doping is larger than $\sim 5 \times 10^{14} \mathrm{~cm}^{-2}$, and eventually reaches zero at $\sim 7 \times 10^{14} \mathrm{~cm}$ ${ }^{2}$. Thereafter, the magnetic moment oscillates between zero and one when the hole density further increases. By comparing the DOS at hole concentrations of $4 \times 10^{14} \mathrm{~cm}^{-}$ ${ }^{2}, 7 \times 10^{14} \mathrm{~cm}^{-2}$ and $8 \times 10^{14} \mathrm{~cm}^{-2}$ in the right panel of Fig. 5 (d), it is evident that the upmost oxygen atoms of the bottom layer $\left(\mathrm{O}_{\mathrm{b}}\right)$ play more and more important roles when the hole concentration is larger than $\sim 4 \times 10^{14} \mathrm{~cm}^{-2}$, as its contribution to the DOS becomes closer to the Fermi level. Thereafter, at $\sim 7 \times 10^{14} \mathrm{~cm}^{-2}$, the DOS contributed by the $\mathrm{O}_{\mathrm{t}}$ and $\mathrm{O}_{\mathrm{b}}$ is merely the same, but with opposite spin direction, which is especially noticeable around the Fermi level. This leads to an antiferromagnetic state in the system. Finally, at $\sim 8 \times 10^{14} \mathrm{~cm}^{-2}$, the DOS contributed respectively by the $\mathrm{O}_{\mathrm{t}}$ and $\mathrm{O}_{\mathrm{b}}$ almost overlap. In this case, the ferromagnetic state is more energetically favorable.

The Curie temperatures $\left(T_{c}\right)$ of the $\mathrm{M}_{2} \mathrm{O}_{3}$ monolayers as well as the $\mathrm{Ga}_{2} \mathrm{O}_{3}$ bilayer were next computed, using Monte Carlo simulations. Based on the 2D Ising model, their spin Hamiltonian is given by

$$
H=-\sum_{<i j>} J_{1} \boldsymbol{S}_{\imath} \cdot \boldsymbol{S}_{j}-\sum_{<<i j>>} J_{2} \boldsymbol{S}_{\imath} \cdot \boldsymbol{S}_{j}
$$

where $\langle i j\rangle$ and $\langle<i j\rangle>$ are the summation over the pairs of nearest and second nearest oxygen atoms, respectively. $J_{1}, J_{2}$ are the nearest-neighbor and second nearest-neighbor exchange interactions, respectively. $\vec{S}_{\imath}$ (or $\boldsymbol{S}_{j}$ ) is a unit vector. For $\mathrm{Ga}_{2} \mathrm{O}_{3}$ monolayer and bilayer, we only considered the nearest-neighbor exchange parameter, since only the topmost oxygen atoms contribute to the magnetic moment, while for $\operatorname{In}_{2} \mathrm{O}_{3}$ monolayer, we considered both exchange parameters, which are derived from the two $\mathrm{O}$ atoms in the same or different planes. These exchange parameters can be evaluated using the energy difference between ferromagnetic and antiferromagnetic phases, obtained from first-principles calculations. Details about the construction of the ferromagnetic and antiferromagnetic configurations, as well as the calculation of the 
exchange parameters are given in Fig. S4 and Fig. S5. The values of the exchange parameters under different hole doping concentrations are summarized in Table S3. It is clear that a higher hole density gives rise to larger exchange parameters.

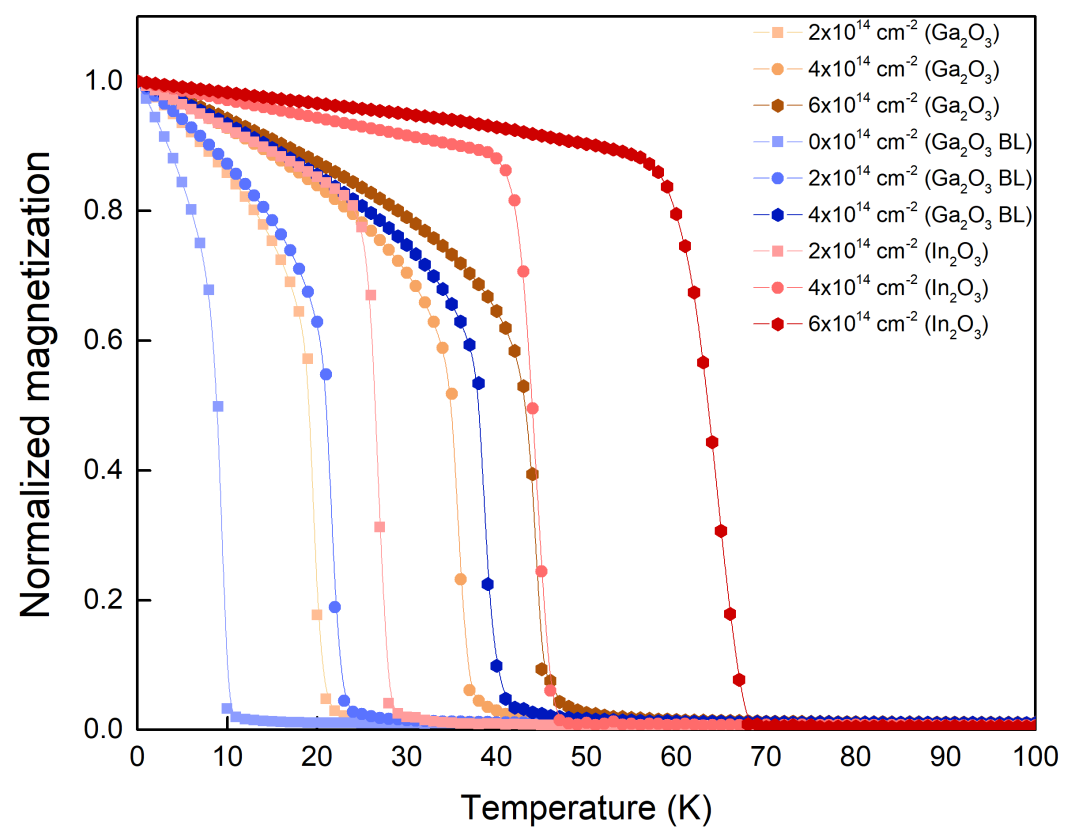

Fig. 6. Monte Carlo simulations of the normalized magnetization of $\mathrm{M}_{2} \mathrm{O}_{3}$ monolayers and $\mathrm{Ga}_{2} \mathrm{O}_{3}$ bilayer (BL) as a function of temperature, for various hole doping densities, as indicated in the figure caption.

The temperature-dependent normalized magnetization curves, computed from Monte Carlo simulations under different hole doping densities, are displayed in Fig. 6. The magnetization remains high in the low temperature range, and then drops to near zero at a certain critical (Curie) temperature. The temperature dependent magnetization is fitted using the Curie-Bloch equation in the classical limit:

$$
\mathrm{m}(T)=\left(1-\frac{T}{T_{c}}\right)^{\beta}
$$

where $T$ is the temperature and $\beta$ is a critical exponent. Obviously, $T_{\mathrm{c}}$ is proportional to 
the exchange interactions and the hole doping concentrations in $\mathrm{M}_{2} \mathrm{O}_{3}$ monolayers and $\mathrm{Ga}_{2} \mathrm{O}_{3}$ bilayer. At the same doping level, the $\mathrm{In}_{2} \mathrm{O}_{3}$ monolayer has the highest $T_{\mathrm{c}}$, followed by the $\mathrm{Ga}_{2} \mathrm{O}_{3}$ bilayer, then by the $\mathrm{Ga}_{2} \mathrm{O}_{3}$ monolayer. At a hole concentration of $\sim 2 \times 10^{14} \mathrm{~cm}^{-2}$, the fitted $T_{\mathrm{c}}$ is $\sim 20 \mathrm{~K} / 24 \mathrm{~K} / 27 \mathrm{~K}$ for $\mathrm{Ga}_{2} \mathrm{O}_{3}$ monolayer $/ \mathrm{Ga}_{2} \mathrm{O}_{3}$ bilayer/ $/ \mathrm{In}_{2} \mathrm{O}_{3}$ monolayer, respectively, while it increases to $36 \mathrm{~K} / 39 \mathrm{~K} / 43 \mathrm{~K}$ when the hole doping density reaches $\sim 4 \times 10^{14} \mathrm{~cm}^{-2}$. At $\sim 6 \times 10^{14} \mathrm{~cm}^{-2}, T_{\mathrm{c}}$ is $45 \mathrm{~K}$ and $62 \mathrm{~K}$ for $\mathrm{Ga}_{2} \mathrm{O}_{3}$ and $\mathrm{In}_{2} \mathrm{O}_{3}$ monolayers, respectively, being comparable to the ones of monolayer $\mathrm{CrI}_{3}(45 \mathrm{~K}),{ }^{16}$ and bilayer $\mathrm{Cr}_{2} \mathrm{Ge}_{2} \mathrm{Te}_{6}(30 \mathrm{~K}) .^{43}$ Although the second nearest-neighbor Deleted: 2D exchange interaction $\left(J_{2}\right)$ is several times larger in $\operatorname{In}_{2} \mathrm{O}_{3}$ as compared to $\mathrm{Ga}_{2} \mathrm{O}_{3}$, their computed Curie temperatures are comparable. It may be due to the "competition" between $J_{1}$ and $J_{2}$, arising from the weaker AFM interaction and stronger FM interaction in the $\mathrm{In}_{2} \mathrm{O}_{3}$ monolayer, leading to a lower $T_{\mathrm{c}}$ than expected.

\section{IV.CONCLUSIONS}

In summary, by combining first-principles calculations with the evolutionary algorithm USPEX, we discovered energetically stable phases of $2 \mathrm{D} \mathrm{Ga}_{2} \mathrm{O}_{3}$ and $\mathrm{In}_{2} \mathrm{O}_{3}$. Calculations of their phonon dispersion curves and ab-initio molecular dynamics simulations confirmed their structural and thermal stability. Both $\mathrm{Ga}_{2} \mathrm{O}_{3}$ and $\operatorname{In}_{2} \mathrm{O}_{3}$ monolayers are predicted to be intrinsically non-magnetic semiconductors, with flat band edges at the top of their valence bands, resulting in sharp peaks in their electronic density of states. A spontaneous ferromagnetic phase transition occurs upon hole doping of these $2 \mathrm{D}$ materials, which turns them into half-metals over a wide range of hole densities.

The bilayer structure of $\mathrm{Ga}_{2} \mathrm{O}_{3}$ was also investigated and it showed ferromagnetism without external hole doping, resulting from the non-centrosymmetric structure of $2 \mathrm{D}$ $\mathrm{Ga}_{2} \mathrm{O}_{3}$. In this case, the spontaneous ferromagnetic order is triggered by the charge transfer taking place between the two layers of the $\mathrm{Ga}_{2} \mathrm{O}_{3}$ bilayer. Using Monte Carlo simulations, based on the Heisenberg model, the estimated Curie temperatures of the $\mathrm{Ga}_{2} \mathrm{O}_{3}$ and $\mathrm{In}_{2} \mathrm{O}_{3}$ monolayers and the $\mathrm{Ga}_{2} \mathrm{O}_{3}$ bilayer are proportional to the hole doping density, and are comparable to the ones of ${ } \mathrm{CrI}_{3}$ monolayer and $2 \mathrm{D} \mathrm{Cr}_{2} \mathrm{Ge}_{2} \mathrm{Te}_{6}$ bilayer 
at moderate hole doping densities. We hope that our work can inspire the synthesis of these new 2D materials and the experimental study of their predicted magnetic properties upon hole doping.

\section{SUPPLEMENTARY MATERIAL}

See the supplementary material for detailed information about the computed hybrid band structures of the $\mathrm{M}_{2} \mathrm{O}_{3}$ monolayers and $\mathrm{Ga}_{2} \mathrm{O}_{3}$ bilayer, variation rates of the bond lengths and bond angles of the charged $\mathrm{M}_{2} \mathrm{O}_{3}$ monolayers after atomic relaxations, schematics of the various magnetic structures, and details about the calculations of the exchange interactions, as well as their computed values.

\section{ACKNOWLEDGMENTS}

Part of this work has been financially supported by the KU Leuven Research Fund, project C14/17/080; Part of the computational resources and services used in this work have been provided by the VSC (Flemish Supercomputer Center), funded by the Research Foundation Flanders (FWO) and the Flemish Government - department EWI.

\section{AVAILABILITY OF DATA}

The data that support the findings of this study are available from the corresponding author upon reasonable request.

\section{REFERENCES}

F. Tang, H. C. Po, A. Vishwanath, and X. Wan, Nature 566, 486 (2019).

2 M. G. Vergniory, L. Elcoro, C. Felser, N. Regnault, B. A. Bernevig, and Z. Wang, Nature 566, 480 (2019).

3 T. Zhang, Y. Jiang, Z. Song, H. Huang, Y. He, Z. Fang, H. Weng, and C. Fang, Nature 566, 475 (2019).

4 A. Jain, Y. Shin, and K. A. Persson, Nat. Rev. Mater. 1, 15004 (2016).

5 N. Mounet, M. Gibertini, P. Schwaller, D. Campi, A. Merkys, A. Marrazzo, T. Sohier, I. E. Castelli, A. Cepellotti, G. Pizzi, and N. Marzari, Nat. Nanotechnol. 13, 246 (2018).

6 S. Haastrup, M. Strange, M. Pandey, T. Deilmann, P. S. Schmidt, N. F. Hinsche, M. N. Gjerding, D. 
Torelli, P. M. Larsen, A. C. Riis-Jensen, J. Gath, K. W. Jacobsen, J. Jørgen Mortensen, T. Olsen, and K. S. Thygesen, 2D Mater. 5, 042002 (2018).

7 H. Zheng, X. Li, N. Chen, S. Xie, W. Q. Tian, Y. Chen, H. Xia, S. B. Zhang, and H. Sun, Phys. Rev. B 92 (2015).

8 A. Molle, J. Goldberger, M. Houssa, Y. Xu, S. C. Zhang, and D. Akinwande, Nat. Mater. 16, 163 (2017).

9 C. Zhang, J. Liu, H. Shen, X. Li, and Q. Sun, Chem. Mater. 29, 8588 (2017).

10 Y. Wang and Y. Ding, J. Mater. Chem. C 6, 2245 (2018).

11 X. Zhang, Z. Yu, S. Wang, S. Guan, H. Y. Yang, Y. Yao, and S. A. Yang, J. Mater. Chem. A 4, 15224 (2016).

12 A. Zunger, Nature 566, 447 (2019).

13 O. I. Malyi, G. M. Dalpian, X. Zhao, Z. Wang, and A. Zunger, Mater. Today 32, 35 (2020).

14 J. Liu, Q. Sun, Y. Kawazoe, and P. Jena, Phys Chem Chem Phys 18, 8777 (2016).

15 B. Huang, G. Clark, D. R. Klein, D. MacNeill, E. Navarro-Moratalla, K. L. Seyler, N. Wilson, M. A. McGuire, D. H. Cobden, D. Xiao, W. Yao, P. Jarillo-Herrero, and X. Xu, Nat Nanotechnol 13, 544 (2018).

16 B. Huang, G. Clark, E. Navarro-Moratalla, D. R. Klein, R. Cheng, K. L. Seyler, D. Zhong, E. Schmidgall, M. A. McGuire, D. H. Cobden, W. Yao, D. Xiao, P. Jarillo-Herrero, and X. Xu, Nature 546, 270 (2017).

17 Z. Wang, T. Zhang, M. Ding, B. Dong, Y. Li, M. Chen, X. Li, J. Huang, H. Wang, X. Zhao, Y. Li, D. Li, C. Jia, L. Sun, H. Guo, Y. Ye, D. Sun, Y. Chen, T. Yang, J. Zhang, S. Ono, Z. Han, and Z. Zhang, Nat Nanotechnol 13, 554 (2018).

18 N. León-Brito, E. D. Bauer, F. Ronning, J. D. Thompson, and R. Movshovich, J. Appl. Phys. 120, 083903 (2016).

19 Z. Fei, B. Huang, P. Malinowski, W. Wang, T. Song, J. Sanchez, W. Yao, D. Xiao, X. Zhu, A. F. May, W. Wu, D. H. Cobden, J. H. Chu, and X. Xu, Nat Mater 17, 778 (2018).

20 W. Xing, Y. Chen, P. M. Odenthal, X. Zhang, W. Yuan, T. Su, Q. Song, T. Wang, J. Zhong, S. Jia, X. C. Xie, Y. Li, and W. Han, 2D Mater. 4, 024009 (2017).

21 Y. Sun, R. C. Xiao, G. T. Lin, R. R. Zhang, L. S. Ling, Z. W. Ma, X. Luo, W. J. Lu, Y. P. Sun, and Z. G. Sheng, Appl. Phys. Lett. 112, 072409 (2018).

22 S. Y. Park, D. S. Kim, Y. Liu, J. Hwang, Y. Kim, W. Kim, J. Y. Kim, C. Petrovic, C. Hwang, S. K. Mo, H. J. Kim, B. C. Min, H. C. Koo, J. Chang, C. Jang, J. W. Choi, and H. Ryu, Nano Lett 20, 95 (2020).

23 F. Zheng, J. Zhao, Z. Liu, M. Li, M. Zhou, S. Zhang, and P. Zhang, Nanoscale 10, 14298 (2018).

24 L. Webster and J.-A. Yan, Phys. Rev. B 98, 144411 (2018).

25 J. Zhang, B. Zhao, T. Zhou, Y. Xue, C. Ma, and Z. Yang, Phys. Rev. B 97, 085401 (2018).

26 K. Iordanidou, M. Houssa, J. Kioseoglou, V. V. Afanas'ev, A. Stesmans, and C. Persson, ACS Appl. Nano Mater. 1, 6656 (2018).

27 M. Houssa, K. Iordanidou, G. Pourtois, V. V. Afanas'ev, and A. Stesmans, AIP Adv. 8, 055010 (2018).

28 T. Cao, Z. Li, and S. G. Louie, Phys. Rev. Lett. 114, 236602 (2015).

29 L. Seixas, A. S. Rodin, A. Carvalho, and A. H. Castro Neto, Phys. Rev. Lett. 116, 206803 (2016).

30 A. O. Lyakhov, A. R. Oganov, H. T. Stokes, and Q. Zhu, Comput. Phys. Commun. 184, 1172 (2013).

31 A. R. Oganov and C. W. Glass, J Chem Phys 124, 244704 (2006).

32 G. Kresse, Phys. Rev. B 59, 1758 (1999). 
33 G. Kresse and F. J, Phys. Rev. B 54, 11169 (1996).

34 J. P. Perdew, K. Burke, and M. Ernzerhof, Phys. Rev. Lett. 77, 3865 (1996).

35 J. Heyd, G. E. Scuseria, and M. Ernzerhof, J Chem Phys 118, 8207 (2003).

36 J. Klimeš, D. R. Bowler, and A. Michaelides, Phys. Rev. B 83, 195131 (2011).

37 A. Togo and I. Tanaka, Scr. Mater. 108, 1 (2015).

38 N. Shuichi, Prog. Theor. Phys. Suppl 103, 1 (1991).

39 N. Shuichi, J Chem Phys 81, 511 (1984)

40 R. F. Evans, W. J. Fan, P. Chureemart, T. A. Ostler, M. O. Ellis, and R. W. Chantrell, J. Phys Condens. Matter 26, 103202 (2014).

41 J. Liu and S. T. Pantelides, 2D Mater. 6, 025001 (2019).

42 R. Peng, Y. Ma, S. Zhang, B. Huang, L. Kou, and Y. Dai, Mater. Horiz. 7, 504 (2020).

43 C. Gong, L. Li, Z. Li, H. Ji, A. Stern, Y. Xia, T. Cao, W. Bao, C. Wang, and Y. J. N. Wang, Nature 546, 265 (2017). 